\title{
Healthcare Fragmentation and the Frequency of Radiology and Other Diagnostic Tests: A Cross-Sectional Study
}

\author{
Lisa M. Kern, $M D, M P H^{1,2}$, Joanna K. Seirup, $M P H^{2}$, Lawrence P. Casalino, $M D, P h D^{2}$, and \\ Monika M. Safford, MD ${ }^{7}$
}

'Division of General Internal Medicine, Department of Medicine, Weill Cornell Medicine, New York, NY, USA; ${ }^{2}$ Division of Health Policy and Economics, Department of Healthcare Policy \& Research, Weill Cornell Medicine, New York, NY, USA.

\begin{abstract}
BACKGROUND: Fragmented ambulatory care has been associated with high rates of emergency department visits and hospitalizations, but effects on other types of utilization are unclear.
\end{abstract}

OBJECTIVE: To determine whether more fragmented care is associated with more radiology and other diagnostic tests, compared to less fragmented care.

DESIGN: We conducted a cross-sectional study using claims from five commercial payers for 2010. The study took place in the Hudson Valley, a seven-county region in New York State.

PARTICIPANTS: We included adult patients who were insured through the participating payers and were attributed to a primary care physician in the region. We restricted the cohort to those with $\geq 4$ ambulatory visits, as measures of fragmentation are not reliable if based on $\leq 3$ visits $(N=126,801)$.

MAIN MEASURES: For each patient, we calculated fragmentation using a reversed Bice-Boxerman Index, which we divided into seven categories. We used negative binomial regression to determine the association between fragmentation category and rates of radiology and other diagnostic tests, stratified by number of chronic conditions and adjusting for patient age, gender, and number of visits.

KEY RESULTS: Patients with the most fragmented care had approximately twice as many radiology and other diagnostic tests as patients with the least fragmented care, across all groups stratified by number of chronic conditions (each adjusted $p<0.0001$ ). For example, among patients with $\geq 5$ chronic conditions, those with the least fragmented care had 258 tests per 100 patients, and those with the most fragmented care had 542 tests per 100 patients $(+284$ tests per 100 patients, or $+110 \%$, adjusted $p<0.0001)$.

CONCLUSION: More fragmented care was independently associated with higher rates of radiology and other diagnostic tests than less fragmented care.

KEY WORDS: ambulatory care; healthcare utilization; radiology.

J Gen Intern Med 32(2):175-81

DOI: $10.1007 /$ s11606-016-3883-Z

(C) Society of General Internal Medicine 2016

Received April 21, 2016

Revised August 25, 2016

Accepted September 22, 2016

Published online October 27, 2016

\section{INTRODUCTION}

Patients with chronic disease often see a multitude of outpatient providers. For example, Medicare beneficiaries see a median of seven physicians in four different practices each year. ${ }^{1}$ Although seeing more than one physician may be clinically appropriate, and may be necessary to achieve recommended care, this dispersion of care creates a challenge. The typical primary care physician has 229 other physicians in 117 practices with whom to coordinate care - for their Medicare beneficiaries alone. ${ }^{2}$ Communication across physicians caring for the same patient is often inconsistent and incomplete, with relevant clinical information missing in one of every seven primary care visits. ${ }^{3}$

Fragmentation of ambulatory care has been associated with higher rates of avoidable emergency department visits and hospitalizations, higher costs, and lower patient satisfaction. $^{4-7}$ The relationship between fragmentation of care and other types of utilization, such as radiology and other diagnostic tests, has not been well studied. Previous studies have also not fully explored the relationship between fragmentation and chronic disease. This is important, because the number of chronic conditions may serve as a potential confounder in the relationship between fragmentation and subsequent outcomes.

We sought to determine the association between fragmentation and the frequency of radiology and other diagnostic tests, with the hypothesis that more fragmentation would be associated with more testing. We specifically sought to determine how such an association might vary with the number of chronic conditions. This question has implications for patients, providers, and policy makers alike, in part because provider reimbursement is shifting from fee-for-service to value-based payments, which makes providers increasingly responsible for all the care that their patients receive (not just care that they themselves provide). ${ }^{8}$

\section{METHODS}

Overview. We conducted a cross-sectional study of commercially insured adults who were attributed to primary care physicians in the Hudson Valley region of New York in 2010. We determined associations between healthcare fragmentation 
and the frequency of radiology and other diagnostic tests, stratifying patients by their numbers of chronic conditions and adjusting for other potential confounders. Institutional review boards at Weill Cornell Medical College and Kingston Hospital approved the protocol.

Setting. The Hudson Valley consists of 7 counties (Dutchess, Orange, Putnam, Rockland, Sullivan, Ulster, and Westchester) north of New York City. Approximately $85 \%$ of people in this region live in urban or suburban areas. ${ }^{9}$ At the time of the study, most physicians in the region were in small- and medium-sized private practices $(\leq 25 \text { physicians per practice })^{10}$ and accepted insurance from multiple payers, with fee-forservice as the dominant model of payment.

Participants. We included adults $(\geq 18$ years old) who had commercial health insurance through one of five participating plans, which together insured approximately $60 \%$ of the region's commercially insured population (Aetna, Capital District Physicians' Health Plan, Hudson Health Plan, MVP Health Care, and UnitedHealthcare). These plans were convened by the Taconic Health Information Network and Community (THINC), a community-based organization that is now part of HealthlinkNY. ${ }^{11}$

Data Source. A third-party company had aggregated claims across payers and attributed patients to primary care physicians in the Hudson Valley, using previously specified logic. ${ }^{12}$ The data set included all claims for 2010 for the attributed patients, regardless of the rendering provider. Variables included patient study ID, patient age, patient gender, date of service, rendering provider study ID, Current Procedural Terminology (CPT) codes, and International Classification of DiseasesNinth Revision (ICD-9) codes. The data set also included patient-level counts of outpatient radiology and other diagnostic tests, which had been derived by the data aggregator through proprietary algorithms in the context of a broader project that attempted to place all utilization into eight categories, with outpatient radiology and other diagnostic tests being one category. ${ }^{13}$ "Other diagnostic tests" included outpatient diagnostic tests that were assisted by imaging but may not have been performed by a radiologist per se, such as cardiac catheterization. This category excluded laboratory tests, such as blood and urine tests.

Variables. We identified ambulatory visits with CPT codes using a modified version of the definition by the National Committee for Quality Assurance. ${ }^{14}$ Modifications restricted the definition to evaluation-and-management visits for adults that would take place in an office setting, excluding managementonly visits (e.g., dialysis, chemotherapy, and physical therapy) and excluding non-office-based visits (e.g., home visits and visits in nursing facilities). This definition also excluded emergency department visits.
We used ICD-9 codes to calculate the total number of chronic conditions for each patient, using the 27 chronic conditions defined by the Centers for Medicare and Medicaid Services Chronic Condition Warehouse. ${ }^{15}$ We combined two dementia categories into one to avoid double-counting, yielding 26 unique categories.

We calculated a fragmentation score for each patient based on the Bice-Boxerman Index (Appendix). ${ }^{16}$ This index was originally labeled as a measure of "continuity," but —as its originators intended - it actually measures more than consistency of care with a single provider. The index captures both "dispersion" (the spread of a patient's care across multiple providers) ${ }^{17}$ and "density" (the relative share of visits by each provider) ${ }^{17}$. This index has been used previously to predict emergency department visits, hospitalizations, and costs of care. ${ }^{4}$ The Bice-Boxerman Index has been found to be highly correlated with other measures of continuity or fragmentation, such as the Herfindahl-Hirschman Index, the Usual Provider of Care Index, and the Sequential Continuity Index. ${ }^{18}$

Values of the original Bice-Boxerman Index range from 0 (least continuity, or most fragmentation) to 1 (most continuity, or least fragmentation). Patients receive a raw score of 0 if each ambulatory visit is with a different provider and a raw score of 1 if all visits are with the same provider. Other patterns of visits and providers receive intermediate scores; patterns with high dispersion (many providers) and low density (a relatively low proportion of visits by each provider) receive worse scores (indicating more fragmentation) than patterns with low dispersion and high density. To facilitate interpretation, we reversed the scores (calculating 1 minus the BiceBoxerman Index score), so that higher values reflected more fragmentation. For the remainder of the paper, we refer to these transformed scores simply as scores.

The theoretical distribution of the Bice-Boxerman Index is skewed rather than normal, because the number of possible patterns that reflect fragmented care are more numerous than the number of possible patterns that reflect continuous care. Thus, we did not treat the index as a continuous variable; instead, we created seven categories of fragmentation scores. Scores of 0.00 (least fragmentation) were placed in category 1 , scores of 1.00 (most fragmentation) were placed in category 7 , and the scores in between were divided into quintiles (categories 2-6). We chose quintiles because they provided a more detailed view than tertiles or quartiles, while still being a manageable number of categories for interpretation.

Statistical Analysis. We excluded patients if all claims for ambulatory visits had missing providers $(<0.5 \%$ of the cohort). We also excluded patients who had outlier observations ( $>99.9$ th percentile) for the number of ambulatory visits or providers, as some of those observations appeared to be erroneous. We used descriptive statistics to characterize patients and ambulatory visits.

We restricted the cohort to patients with $\geq 4$ ambulatory visits, because measures of fragmentation may not be reliable 
if based on $\leq 3$ visits. ${ }^{6}$ We had complete data on patient characteristics and visit patterns for $>99.9 \%$ of the patients in this cohort. We stratified patients by fragmentation category and summarized utilization of ambulatory visits within category. We reported unadjusted mean counts of radiology and other diagnostic tests per 100 patients, stratified by fragmentation category and by the number of chronic conditions $(0,1-2,3-4$, $\geq 5$ ). To determine adjusted rates of radiology and other diagnostic tests, we used the exponentiated least squares means by fragmentation category from generalized linear models with a negative binomial distribution. This approach was chosen because the data were non-negative and over-dispersed. Models were stratified by chronic condition group and adjusted for age, gender, and number of ambulatory visits.

Statistical analyses were conducted with SAS statistical software (version 9.4; SAS Institute Inc., Cary, NC).

\section{RESULTS}

Study sample. In 2010, there were 190,223 adults in the Hudson Valley who were insured by a participating health plan, were attributed to a primary care physician, and had $\geq 1$ claim (Fig. 1). Of those, 189,429 (99.6\%) had $\geq 1$ ambulatory visit with a non-missing rendering provider. We excluded 288 adults $(0.2 \%)$ with outlier observations for visits or providers, leaving 189,141 patients. Of those, 62,340 (33\%) had $\leq 3$ visits, and 126,801 (67\%) had $\geq 4$ ambulatory visits.

Patient characteristics. Patients with $\leq 3$ visits had a mean age of 41.8 years, fewer than half ( $47.5 \%$ ) were female, and they had a median of 0 chronic conditions (Table 1).

Patients with $\geq 4$ visits were included in subsequent analyses. They were older, with a mean age of 51.2 years (Table 1 ). More than $75 \%$ were younger than age 65; the maximum age was $>100$ years. More than half were female $(61.8 \%)$. The median number of chronic conditions was two. The distribution of the number of chronic conditions was as follows: $18 \%$ of patients had zero chronic conditions, $35 \%$ had one or two, $28 \%$ had three or four, and $19 \%$ had five or more. Patients had a median of eight ambulatory visits (range 4 to 56), with a median of four unique providers (range 1 to 17). The typical patient had $44 \%$ of visits with the most frequently seen provider (range $9 \%$ to $100 \%$ ).

Healthcare fragmentation. Fragmentation scores were not determined exclusively by the number of visits (Table 2). Patients with more fragmented care generally saw more providers than patients with less fragmented care. Patients with more fragmented care had a lower proportion of their visits with the most frequently seen provider than patients with less fragmented care.
Healthcare fragmentation and radiology utilization. As fragmentation increased, utilization of radiology and other diagnostic tests also increased, adjusting for patient age, gender, and number of ambulatory visits (Fig. 2). This association was observed within each stratum of chronic conditions. Patients with the most fragmented care had approximately twice as many radiology and other diagnostic tests as patients with the least fragmented care, regardless of their number of chronic diseases. Because patients with a greater number of chronic conditions tended to have more radiology and other diagnostic tests than patients with fewer chronic conditions, a doubling of the number of tests had the largest absolute effect for those with the most chronic conditions.

For example, in adjusted analyses, patients with 0 chronic conditions and the least fragmented care used 71 tests per 100 patients (Fig. 2). Patients with 0 chronic conditions and the most fragmented care used 139 tests per 100 patients, equivalent to an absolute difference of 68 tests per 100 patients, or a relative increase of $+95 \%$ for category 7 vs. category 1 (adjusted $p<0.0001$ ). Patients with $\geq 5$ chronic conditions used 258 tests per 100 patients if they had the least fragmented care and 542 tests per 100 patients if they had the most fragmented care ( +284 tests per 100 patients, $+110 \%$, adjusted $p<0.0001)$.

\section{DISCUSSION}

In this study of commercially insured adults, patients with the most fragmented care had twice as many radiology and other diagnostic tests as patients with the least fragmented care, regardless of the number of chronic conditions. Because patients with more chronic conditions had more tests than those with fewer chronic conditions, a doubling of the number of tests had the largest absolute effect for those with the most chronic conditions. These associations were observed adjusting for patient age, gender, and number of ambulatory visits.

We are aware of only two other studies that have explored the effect of fragmentation on radiology testing. In one study of Medicare beneficiaries, seeing a greater number of physicians in the year following a stroke was associated with a higher likelihood of having $\geq 4$ computerized tomography (CT) scans of the head that year, compared to seeing fewer physicians. ${ }^{19}$ That study was limited by its ecologic design, with the numbers of physicians and CT scans calculated at the level of the hospital referral region rather than the patient level. Another study considered the relationship between fragmentation and rates of use for 19 "potentially overused" procedures among Medicare beneficiaries. ${ }^{20}$ Greater fragmentation was associated with more testing for five of eight radiology tests considered. ${ }^{20}$ That study adjusted for the number of chronic diseases rather than stratify by number of chronic diseases. 


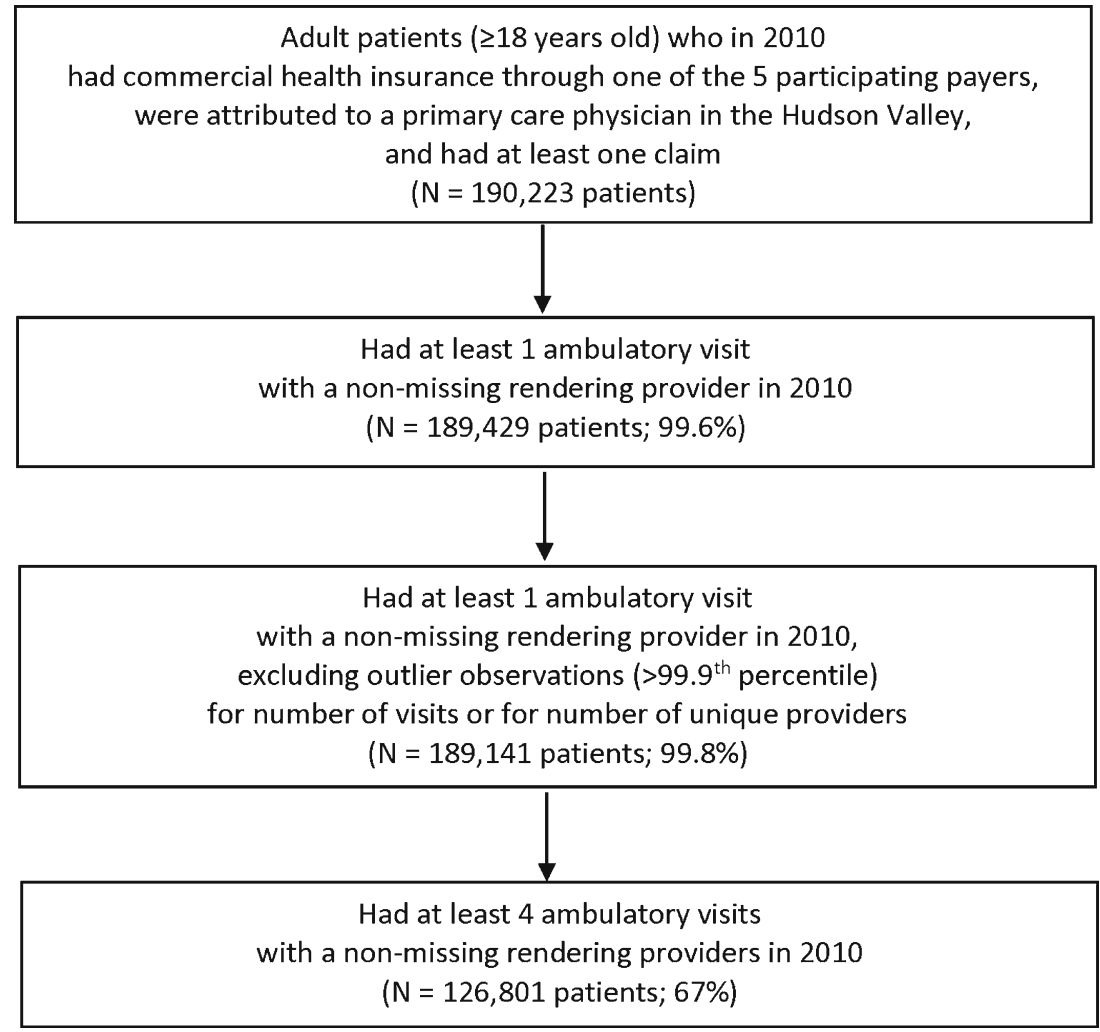

Figure 1 Derivation of the study sample.

The rates of testing we observed were somewhat higher than rates of testing observed elsewhere, although few such studies are available for comparison. A study in Group Health in Seattle, WA, found that, on average, between 2000 and 2006, patients aged 45-54 years had approximately 1400 diagnostic imaging tests per 1000 enrollees per year, and patients aged 55-64 years had approximately 1900 diagnostic imaging tests per 1000 enrollees per year. ${ }^{21}$ That study confirms that having multiple tests per patient per year is common; however, it did not restrict its cohort to those with $\geq 4$ visits per year and did not stratify by the number of chronic conditions, thereby precluding precise comparisons. Another study, in six integrated health systems across the country, found that rates of diagnostic medical imaging increased nearly every year from 1996 to
$2010,{ }^{22}$ suggesting that the rates of testing observed in the first study above would be even higher in 2010, the year corresponding to our study.

Our study adds to the literature in several ways. First, it extends observations of fragmentation - most of which have been made previously in Medicare populations - to a younger, commercially insured population. This is relevant, because patterns of care that patients become accustomed to as adults (ages 18 to 64 years) may contribute to the patterns of care that they seek out as older adults (ages 65 years and older). Second, this study uses stratification to separate the effect of chronic conditions from the effect of fragmentation. Previous studies have typically either considered patients with a specific disease (such as diabetes) ${ }^{4,5}$ or considered all patients together. ${ }^{6}$ Third, this study does not treat fragmentation as a continuous

Table 1 Characteristics of Patients and Their Ambulatory Visits over a One-Year Period

\begin{tabular}{|c|c|c|c|c|}
\hline Patient characteristics & $\begin{array}{l}\text { Total } \\
(N=189,141)\end{array}$ & $\begin{array}{l}\text { Patients with } \\
\leq 3 \text { visits } \\
(N=62,340)\end{array}$ & $\begin{array}{l}\text { Patients with } \\
\geq 4 \text { visits* } \\
(N=126,801)\end{array}$ & $p$ value \\
\hline ge, mean, years $(\mathrm{SD})$ & $48.1(17.2)$ & $41.8(15.9)$ & $51.2(17.0)$ & $<0.001$ \\
\hline Gender, female, \% & 57.1 & 47.5 & 61.8 & $<0.001$ \\
\hline Number of chronic conditions, median (range) & $2(0-16)$ & $0(0-14)$ & $2(0-16)$ & $<0.001$ \\
\hline Number of ambulatory visits per patient, median ( & $5(1-56)$ & $2(1-3)$ & $8(4-56)$ & $<0.001$ \\
\hline Number of unique ambulatory providers per patient, median & $3(1-17)$ & $2(1-3)$ & $4(1-17)$ & $<0.001$ \\
\hline $\begin{array}{l}\text { Proportion of visits with the most frequently seen ambulatory provider, } \\
\text { median (range) }\end{array}$ & $0.50(0.09-1.00)$ & $0.67(0.33-1.00)$ & $0.44(0.09-1.00)$ & $<0.001$ \\
\hline
\end{tabular}

*Only those patients with $\geq 4$ visits were included in the subsequent analyses. $p$ values are based on $t$ tests for continuous variables and chi-square tests for categorical variables. Missing data: gender $(N=29)$, number of chronic conditions $(N=22)$ 
Table 2 Dividing Patients with Four or More Visits into Seven Groups, Based on the Extent of Healthcare Fragmentation

\begin{tabular}{|c|c|c|c|c|c|c|c|}
\hline & $\begin{array}{l}\text { Fragmentation } \\
\text { category, based on } \\
\text { reversed Bice- } \\
\text { Boxerman Index } \\
\text { scores* }\end{array}$ & $\begin{array}{l}\text { Reversed } \\
\text { Bice- } \\
\text { Boxerman In- } \\
\text { dex score } \\
\text { range }\end{array}$ & $\begin{array}{l}\text { Number } \\
\text { of } \\
\text { patients }\end{array}$ & $\begin{array}{l}\text { Number of } \\
\text { chronic } \\
\text { conditions, } \\
\text { median (IQR) }\end{array}$ & $\begin{array}{l}\text { Ambulatory } \\
\text { visits, median } \\
\text { (IQR) }\end{array}$ & $\begin{array}{l}\text { Unique } \\
\text { ambulatory } \\
\text { providers, } \\
\text { median (IQR) }\end{array}$ & $\begin{array}{l}\text { Proportion of } \\
\text { visits with most } \\
\text { frequently seen } \\
\text { provider, } \\
\text { median (IQR) }\end{array}$ \\
\hline \multirow{2}{*}{$\begin{array}{l}\text { Least } \\
\text { fragmented } \\
\text { care }\end{array}$} & 1 & 0.00 & 4614 & $2(1-3)$ & $5(4-6)$ & $1(1-1)$ & $1.00(1.00-1.00)$ \\
\hline & $\begin{array}{l}2 \\
3 \\
4 \\
5 \\
6\end{array}$ & $\begin{array}{l}0.01 \text { to } 0.60 \\
0.60 \text { to } 0.75 \\
0.75 \text { to } 0.83 \\
0.83 \text { to } 0.88 \\
0.88 \text { to } 0.99\end{array}$ & $\begin{array}{l}23,972 \\
22,685 \\
22,756 \\
23,763 \\
23,478\end{array}$ & $\begin{array}{l}2(1-4) \\
3(1-4) \\
3(1-4) \\
2(1-4) \\
2(1-4)\end{array}$ & $\begin{array}{l}6(5-10) \\
8(6-12) \\
9(7-14) \\
8(4-13) \\
9(6-13)\end{array}$ & $\begin{array}{l}2(2-3) \\
3(3-4) \\
4(4-6) \\
5(3-7) \\
6(5-8)\end{array}$ & $\begin{array}{l}0.75(0.67-0.80) \\
0.54(0.50-0.60) \\
0.42(0.38-0.44) \\
0.36(0.31-0.50) \\
0.29(0.23-0.33)\end{array}$ \\
\hline $\begin{array}{l}\text { Most } \\
\text { fragmented } \\
\text { care }\end{array}$ & 7 & 1.00 & 5530 & $1(0-2)$ & $4(4-5)$ & $4(4-5)$ & $0.25(0.20-0.25)$ \\
\hline
\end{tabular}

*These categories were derived from Bice-Boxerman Index scores that had been reversed (equal to 1 minus the raw Bice-Boxerman score). Patients with reversed scores of 0.00 were placed into category 1 , patients with reversed scores of 1.00 were placed into category 7 , and patients with reversed scores of 0.01 to 0.99 were divided into quintiles

$I Q R=$ interquartile range

variable; rather, it recognizes that it is more common for patients to have fragmented care than not, and it uses categories of scores to improve detection of trends.

One mechanism by which fragmented care may lead to more utilization is through providers' lack of access to results of previous radiology tests that may have been ordered by other providers caring for the same patient. If providers cannot access previous results, they may repeat tests. Our study's findings are consistent with this hypothesis, although we did not have detailed information on physician decision making. A previous study found that if physicians accessed radiology test results through an electronic health information exchange, the adjusted odds of repeat testing within 90 days of the original test decreased by $25 \%$ compared to the odds of testing when results were available but not accessed. ${ }^{23}$
This study has several limitations. First, it is cross-sectional and cannot prove causality. Second, the study does not capture the appropriateness of the tests ordered. It is possible that the tests are medically appropriate and not duplicative or unnecessary. For example, some additional testing may occur when patients seek second opinions, which might be appropriate, and which we cannot capture. Because our study does not measure clinical outcomes, we cannot determine whether the additional radiology tests in the groups with more fragmented care had any clinical benefit or harm. Third, although we stratified by chronic conditions, unmeasured differences in severity of illness across subgroups may persist. Other unmeasured confounders may also be present. Fourth, this study took place in one region. This region may be different from other regions of the country, but it may also be similar to other

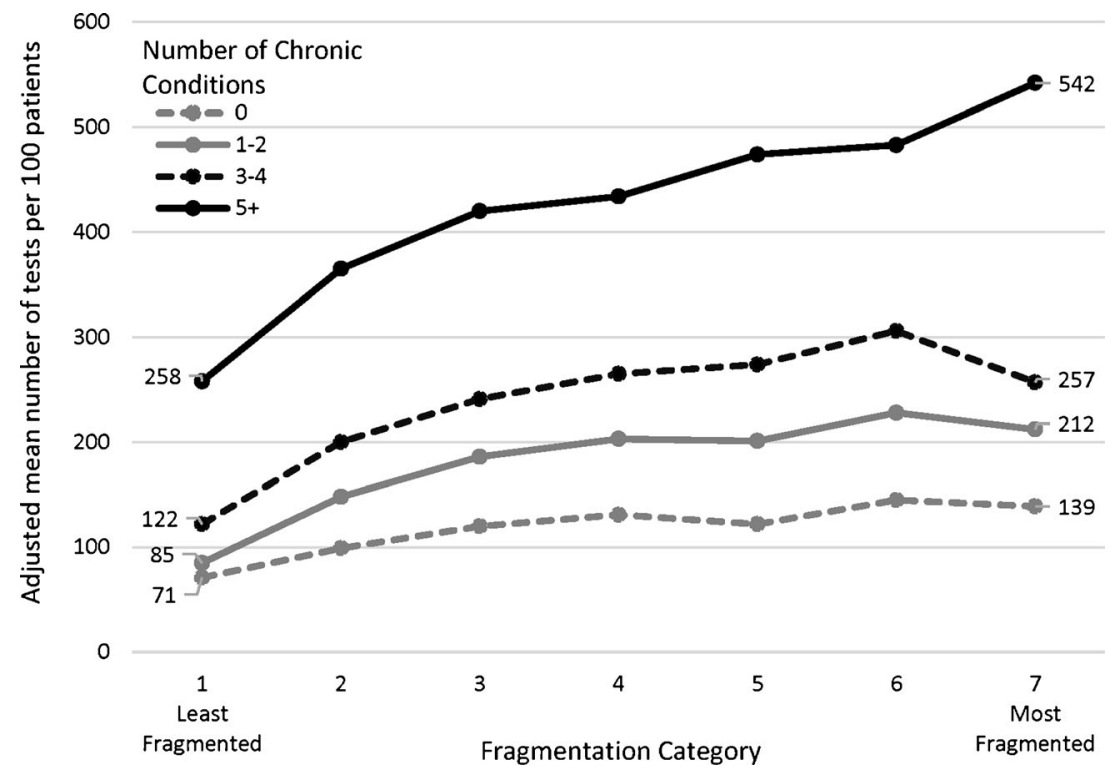

Figure 2 Adjusted mean number of radiology and other diagnostic tests per 100 patients, stratified by fragmentation category and by number of chronic conditions. Note: This analysis is among those patients with $\geq 4$ visits. Fragmentation categories were derived from Bice-Boxerman Index scores that had been reversed (equal to 1 minus the raw Bice-Boxerman score) and divided into seven groups, as described in Table 2. Adjusted means were derived from negative binomial regression models, adjusted for patient age, gender, and number of visits. 
communities with physicians in predominantly small and medium-sized practices accepting multiple types of insurance.

Results from this study raise additional questions that could be explored in future studies, such as how fragmentation affects specific types of radiology tests, how the distribution of visits between generalists and specialists affects the frequency of testing, whether the association between fragmentation and testing varies with particular chronic conditions, and whether the association between fragmentation and testing varies with particular types of health insurance products.

If at least some of the testing that occurs in the context of highly fragmented care represents overuse, then the results of this study suggest that many patients are being exposed to unnecessary radiation, other potential side effects of the tests (such as renal toxicity from contrast dye), and the risk of downstream testing to investigate incidental findings. The cost to patients of a given radiology test varies widely, depending on the provider and the payer; for example, the cost of a single magnetic resonance imaging test can range from approximately $\$ 600$ to more than $\$ 5000$ (not including the cost of the radiologist's reading of the image). ${ }^{24}$ Thus, the potential impact of a twofold difference in radiology utilization could be substantial.

The findings of this study are important for both providers and policymakers, because physician reimbursement is changing. In accountable care organizations and other types of alternative payment models, providers take clinical and financial responsibility for all of a patient's care, even care provided by others. ${ }^{8}$ The Centers for Medicare and Medicaid Services has set a goal of having $50 \%$ of Medicare payments tied to quality or value through alternative payments by the end of 2018 , up from essentially $0 \%$ of payments in $2012 .^{8}$ To this end, value-based payments are a central feature of the Medicare Access and CHIP Reauthorization Act of 2015 (MACRA). ${ }^{25}$

In conclusion, greater fragmentation of care was associated with higher utilization of radiology and other diagnostic tests. Patients with the most fragmented care had approximately twice as many radiology and other diagnostic tests as patients with the least fragmented care, regardless of the number of chronic conditions, and independent of the number of ambulatory visits. Because patients with more chronic conditions had more tests than patients with fewer chronic conditions, fragmentation had the greatest absolute effect for patients with the most $(\geq 5)$ chronic conditions. This suggests that diffuse patterns of care may result in excess testing and avoidable costs. In an era when efficient use of healthcare resources is valued, fragmentation of care may represent an underrecognized source of inefficiency.

Acknowledgments: The authors gratefully acknowledge Susan Stuard, MBA, of HealthlinkNY for facilitating access to the data and for encouraging this evaluation. The authors also thank Phyllis Johnson, MBA, for her assistance with data management.
Corresponding Author: Lisa M. Kern, MD, MPH; Division of Health Policy and Economics, Department of Healthcare Policy \& ResearchWeill Cornell Medicine, New York, NY, USA (e-mail:lmk2003@med.cornell.edu).

Compliance with Ethical Standards: Institutional review boards at Weill Cornell Medical College and Kingston Hospital approved the protocol.

Conflict of Interest: The authors declare that they do not have a conflict of interest.

Funders: This study was funded by the Commonwealth Fund (grant \#20140960), which supported efforts by Dr. Kern and Ms. Seirup. The funding source had no role in the study design, conduct, or reporting. Dr. Kern and Ms. Seirup had full access to all the data in the study and take responsibility for the integrity of the data and the accuracy of the data analysis.

Presentations: This work was presented at the national meeting of the Society of General Internal Medicine on May 13, 2016.

\section{REFERENCES}

1. Pham HH, Schrag D, O'Malley AS, Wu B, Bach PB. Care patterns in Medicare and their implications for pay for performance. N Engl $\mathrm{J}$ Med. 2007;356:1130-9.

2. Pham HH, O'Malley AS, Bach PB, Saiontz-Martinez C, Schrag D. Primary care physicians' links to other physicians through Medicare patients: the scope of care coordination. Ann Intern Med. 2009;150:23642.

3. Smith PC, Araya-Guerra R, Bublitz C, et al. Missing clinical information during primary care visits. JAMA. 2005;293:565-71.

4. Hussey PS, Schneider EC, Rudin RS, Fox DS, Lai J, Pollack CE. Continuity and the costs of care for chronic disease. JAMA Intern Med. 2014; 174:742-8.

5. Liu CW, Einstadter D, Cebul RD. Care fragmentation and emergency department use among complex patients with diabetes. Am J Manag Care. 2010;16:413-20.

6. Nyweide DJ, Anthony DL, Bynum JP, et al. Continuity of care and the risk of preventable hospitalization in older adults. JAMA Intern Med. 2013; 173:1879-85.

7. van Walraven $\mathbf{C}$, Oake $\mathbf{N}$, Jennings A, Forster AJ. The association between continuity of care and outcomes: a systematic and critical review. J Eval Clin Pract. 2010;16:947-56.

8. Burwell SM. Setting value-based payment goals-HHS efforts to improve U.S. health care. N Engl J Med. 2015;372:897-9.

9. U.S. Census Bureau. QuickFacts. (Accessed October 5, 2016, at www. census.gov/quickfacts/.)

10. Stuard SS, Blair AJ. Interval examination: regional transformation of care delivery in the Hudson Valley. J Gen Intern Med. 2011;26:1371-3.

11. HealthlinkNY. About HealthlinkNY. (Accessed August 19, 2016, at http:// www.healthlinkny.com/about-healthlinkny-pg.html.)

12. Kern LM, Edwards A, Kaushal R. The patient-centered medical home, electronic health records, and quality of care. Ann Intern Med. 2014; 160:741-9.

13. Kern LM, Edwards A, Kaushal R. The patient-centered medical home and associations with healthcare quality and utilization: A five-year cohort study. Ann Intern Med. 2016;164:395-405.

14. National Committee for Quality Assurance. HEDIS Volume 2: Technical Specifications, 2015. (Accessed August 19, 2016, at http:// www.ncqa.org/HEDISQualityMeasurement/HEDISMeasures / HEDIS2015.aspx.)

15. Centers for Medicare and Medicaid Services. Chronic Conditions Data Warehouse. (Accessed August 19, 2016, at https://www.ccwdata.org.)

16. Bice TW, Boxerman SB. A quantitative measure of continuity of care. Med Care. 1977;15:347-9.

17. Jee SH, Cabana MD. Indices for continuity of care: a systematic review of the literature. Med Care Res Rev. 2006;63:158-88.

18. Pollack CE, Hussey PS, Rudin RS, Fox DS, Lai J, Schneider EC. Measuring Care Continuity: A Comparison of Claims-based Methods. Med Care. 2016;54(5):e30-4.

19. Bekelis K, Roberts DW, Zhou W, Skinner JS. Fragmentation of care and the use of head computed tomography in patients with ischemic stroke. Circ Cardiovasc Qual Outcomes. 2014;7:430-6. 
20. Romano MJ, Segal JB, Pollack CE. The association between continuity of care and the overuse of medical procedures. JAMA Intern Med. 2015; 175:1148-54.

21. Smith-Bindman R, Miglioretti DL, Larson EB. Rising use of diagnostic medical imaging in a large integrated health system. Health Aff (Millwood). 2008;27:1491-502.

22. Smith-Bindman R, Miglioretti DL, Johnson E, et al. Use of diagnostic imaging studies and associated radiation exposure for patients enrolled in large integrated health care systems, 1996-2010. JAMA. 2012;307:2400-

23. Vest JR, Kaushal R, Silver M, Hentel K, Kern LM. Health information exchange and the frequency of repeat medical imaging. Am J Manag Care. 2014;11 Spec No. 17:eSP16-eSP24.

24. Bebinger M. How much for an MRI? \$500? \$5,000? A reporter struggles to find out. Kaiser Health News. (Accessed August 19, 2016, at http://khn.org/news/mri-cost-price-comparison-health-insurance/.)

25. Doherty RB. Goodbye, Sustainable Growth Rate - Hello, Merit-Based Incentive Payment System. Ann Intern Med. 2015;163:138-9.

\section{APPENDIX. FORMULA FOR THE ORIGINAL BICE- BOXERMAN INDEX (BBI)*16}

$B B I=\frac{\left(\sum_{i=1}^{p} n_{i}^{2}\right)-n}{n(n-1)}$

where $n=$ total number of visits in the 12-month period, $n_{\mathrm{i}}=$ number of visits to provider $i$, and $p=$ total number of providers

* Note that we used a modified Bice-Boxerman Index (reversed and divided into 7 categories), as described in the "Methods" section. 